\title{
Applied CATIA Secondary Development to Parametric Design of Active Magnetic Bearing
}

\author{
Zhang $\mathrm{Li}^{1}$, Yu Meiyun ${ }^{1}$ and Ren Yafeng ${ }^{2}$ \\ ${ }^{1}$ Qingan group co., LTD., 710077 Xian, China \\ ${ }^{2}$ Northwestern Polytechnical University, 710072 Xian, China
}

\begin{abstract}
Based on the properties of active magnetic bearing, the electromagnetic parameters and structure parameters are analyzed, parametric design method is introduced to study the structure of active magnetic bearing. Through a program personalization process that is in accordance with active magnetic bearing is established. Personalization process aims to build the parametric model of active magnetic bearings and component library by use of CATIA secondary development. Component library is to build assembly model for a multiple degree of freedom magnetic bearing system. Parametric design is a method that provides the direction for its structural design.
\end{abstract}

\section{Introduction}

Active Magnetic Bearing (AMB) is a typical mechatronics system. Due to no contact, wear and lubrication, controlling its dynamic behavior and other series of characteristics [1], the limitation of traditional mechanical bearing has been breakthrough, it appeared that has been widely attention. The structure of an AMB is complex, the performance is synthetically influenced by structure parameters, electromagnetic parameters and control parameters [2], it also provides the reference for the structure design of other parts. Especially for small machinery, the miniaturization of structure is even more important. Therefore, AMB design has been in an important position.

Parametric design is a kind of intelligent design method, defined in advance a series of parameters and the component configuration is driven through parameterized drawing and parametric changing engine. When parametric model is established, it can be easily modified and optimized on structure by designers, and as a result, the design efficiency and design quality is greatly improved. At the same time, it also provides basis on the establishment of component library and assembly system.

\section{Active magnetic bearing}

\subsection{Structure of radial AMB}

The structure of radial AMB is similar with the motor, also divided into two parts: stator and rotor. The stator is composed of electromagnet and coil. Soft magnetic materials are used in the electromagnet. Silicon steel sheet is widely used in radial AMB the same as the rotor so that forms a magnetic circuit loop. Type of the stator slots includes trapezoidal groove, rectangular tank, circular groove. Difference type leads to different functions. For example the radial size that has a trapezoidal groove is small, the transition is smooth; for circular groove the radial size is big, the filling coefficient is high. So, the circular groove is the best choice [3], as shown in Fig. 1.

At the same time, In order to optimize the bearing geometry we distribute the space available in the bearing magnet optimally between iron and copper for maximum load capacity [4], Optimization can already be achieved.

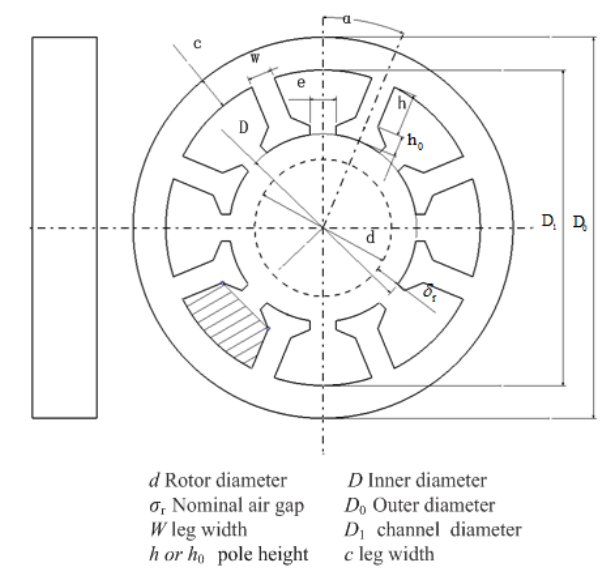

Figure 1. Typical geometry of the radial magnet bearing.

\subsection{Calculating parameters}

Seen from Fig. 1 the structure parameters of the radial magnetic bearing are rotor diameter $d$, nominal air gap $\sigma_{\mathrm{r}}$, Inner diameter $D$, outer diameter $D_{0}$, leg width $w$, pole height $h$ etc. Some parameters are mutual influence such 
as the distance between the poles e is about $10 \sim 20$ times of air gap. The rotor parameters of motor determine the rotor diameter.

The value of nominal air gap associates with the rotor diameter $d$. From practical experience, when $d$ is less than or equal to $100 \mathrm{~mm}, \sigma_{\mathrm{r}}$ is equal to between $0.2 \mathrm{~mm}$ and $0.6 \mathrm{~mm}$; When $d$ is greater than $100 \mathrm{~mm}$, however, the air gap between $0.6 \mathrm{~mm}$ and $1.2 \mathrm{~mm}$. Based on the geometric relationship we can get the inner diameter $D$, the bottom of the channel diameter $D_{1}$, stator diameter $D_{0}$ as shown in formula 1 .

$$
\left\{\begin{array}{c}
D=d+2 \delta_{r} \\
D_{1}=D+2\left(h+h_{0}\right) \\
D_{0}=D_{1}+2 c
\end{array}\right.
$$

If the electromagnetic parameters of the maximum magnetic density $B_{\max }$ and coil current $I_{\max }$ are identified, we can deduce coil slot area $A_{\text {cu }}$ such as formula 2 , said the shadows in the Fig. 1.

$$
\left\{\begin{aligned}
A_{c u} & =\frac{\pi N_{p} d_{w}^{2}}{4 \lambda} \\
d_{w} & =\sqrt{\frac{I_{\max }}{\pi \varepsilon_{0}}} \\
B_{\max } & =\mu_{0} \frac{n I_{\max }}{2 \delta_{r}}
\end{aligned}\right.
$$

where $\lambda$ is the filling coefficient, average value is between 0.5 and $0.7, d_{\mathrm{w}}$ is the coil diameter. $\varepsilon_{0}$ is the coefficient of the sectional area and current. Type 3 is simultaneous deduced by type 2 .

$$
A_{c u}=\frac{B_{\max } \delta_{r}}{2 \lambda \mu_{0} \varepsilon_{0}}
$$

From the formula 3 when $\sigma_{\mathrm{r}}$ and $B_{\max }$ is determined, the coil groove area will be also determined. Therefore, according to the geometric relation we get the formula 4 , deducing the pole height $h$. Generally, magnetic pole height $h_{0}$ is between 0.1 to 0.3 times of $h$.

$$
A_{c u}=\alpha h\left(D_{1}-h\right)-h w
$$

Leg width $w$ is identified based on pole shoe arc length $\left(l=\pi D / N_{\mathrm{p}}-e\right)$, that $w$ is equal to 0.5 to 0.8 times of l. $N_{\mathrm{p}}$ is the number of a column in active magnetic bearing. Static load capacity of an AMB $F_{\max }$ is shown in formula 5 .

$$
F_{\max }=\frac{B_{\max }^{2} A_{a} \cos \alpha}{\mu_{0}}
$$

The magnetic space $A_{\mathrm{a}}$ is shown in formula $6 . K_{\mathrm{s}}$ is the stator stack loading coefficient. $L$ is bearing width (magnetically active). Table 1 shows some basic

\begin{tabular}{|c|c|c|c|c|c|c|c|c|c|}
\hline & $\begin{array}{l}d \\
\mathrm{~nm}\end{array}$ & $\begin{array}{c}d_{\mathrm{i}} \\
\mathrm{mm}\end{array}$ & $\begin{array}{c}D_{0} \\
\mathrm{~mm}\end{array}$ & $\begin{array}{c}\sigma_{\mathrm{r}} \\
\mathrm{mm}\end{array}$ & $\begin{array}{c}L_{\mathrm{t}}-L \\
\mathrm{~mm}\end{array}$ & $\begin{array}{l}N_{\max } \\
\mathrm{r} / \min \end{array}$ & $\begin{array}{c}F_{0} \\
\mathrm{~N} / \mathrm{mm} / \mathrm{T}^{2}\end{array}$ & $N_{\mathrm{p}}$ & \\
\hline & 40 & 24 & 98 & 0.3 & 11 & 900 & 3.9 & 8 & \\
\hline & 60 & 36 & 116 & 0.3 & 12 & 600 & 5.9 & 8 & \\
\hline & 80 & 49 & 138 & 0.4 & 16 & 450 & 7.8 & 8 & \\
\hline & 100 & 74 & 164 & 0.6 & 21 & 360 & 9.8 & 1 & \\
\hline & 120 & 89 & 191 & 0.8 & 25.5 & 300 & 11.8 & 16 & \\
\hline $\begin{array}{c}\text { Analysis of the } \\
\text { design indexes } \\
\text { and modeling }\end{array}$ & $\rightarrow c$ & $\begin{array}{c}\text { Parameter } \\
\text { calculation and } \\
\text { adjustment }\end{array}$ & & & $\begin{array}{c}\text { Par } \\
\text { inp }\end{array}$ & $\begin{array}{l}\text { leter } \\
\text { file }\end{array}$ & $\begin{array}{c}\text { Based on CATIA } \\
\text { parameterization } \\
\text { modeling }\end{array}$ & 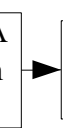 & $\begin{array}{c}\text { component } \\
\text { library }\end{array}$ \\
\hline
\end{tabular}
parameters of the radial magnetic bearing.

$$
A_{a}=K_{s} w L
$$

Table 1. Typical dimensions of radial AMB.

Figure 2. Flow charts of the parametric design on active magnetic bearing.

\section{Parameterized design method of active magnetic bearing}

\subsection{Parametric technology}

Parametric technology is one of the core technology of modern $\mathrm{CAD} / \mathrm{CAE}$ system software. It is also a main research direction in the field of CAD software. In fact, parametric design is to realize the driving of the model by modifying the components parameters that govern the size or assembly relations or change the size of the part or some parts in advance [5].

\subsection{Parametric design of AMB}

Designers get a series of defined parameters through the analysis of the structure and the decomposition of magnetic bearing design indicators. Generally, parametric design process includes the analysis of design indexes and modeling, parameter calculation and adjustment, based on CATIA parameterization modeling, component 
library and so on, as shown in Fig. 2. The analysis of the design indexes and modeling, parameter calculation and adjustment are the core part of the design process, the latter is belong to the parametric design software implementation. And the assembly design of magnetic bearing system can be done by component library.

Analysis of the design indexes and modeling is based on the overall layout, the overall structure and so on. Design indexes include highest speed of rotor, maximum power, radial and axial maximum cutting force, selection of motor parameters, processing and manufacturing features, etc. Parameter calculation and adjustment is designed to estimate the interrelationship of electromagnetic parameters, structural parameters and optimize the parameters that do not meet the requirements of design and size. All of design parameters are outputted into standard format such as txt, $\mathrm{xml}$ file.

An interface of parameter data and the modeling that can quickly complete parametric modeling of magnetic bearing is designed. According to the modern database technology, we have established some component library to complete parameter assembly and the traceability of data information. Component library includes some parts library of a magnetic bearing system such as a rotor shaft library, a radial AMB library and an axial AMB library.

\section{Realization of parametric design}

For development of the parametric design in an AMB, CATIA software platform is introduced, Mainly based on the following consideration:

(1) Parametric design technology is very suitable for designing the shape of the same structure and only the change of size parameters on parts, and AMB just meet this characteristic.

(2) VB or VBA is used for secondary development of CATIA, CATIA provides powerful variable drive and parametric ability in order to achieve the parameters of the design goals, the process is that it provides the corresponding code drive to realize parameterized modeling by calling the underlying CATIA API interface function. Compared with the internal module development, this kind of way is more development depth.

(3) CATIA system is an open and wide application, particularly in the field of aviation and space flight.

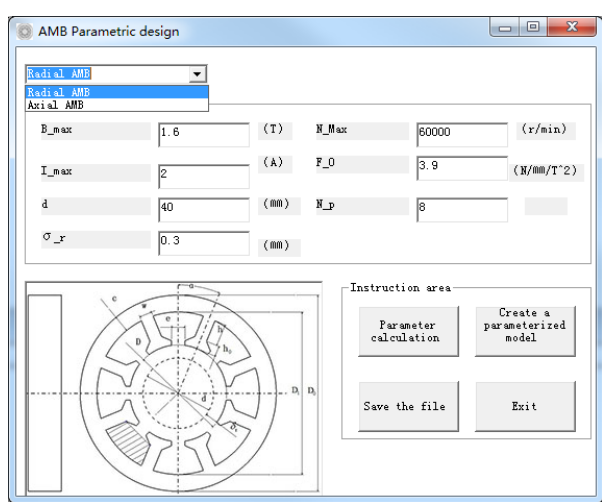

Figure 3. Flow charts of the parametric design on active magnetic bearing.
The development of this system uses Visual Basic 6.0 namely the Automation technology, use of COM technology and interface may realize to invoke the internal module of CATIA. Parametric design system framework and interface in VB is customized, as shown in Fig. 3.

Below is part of the code that is used to invoke internal module of CATIA, analyze and calculate the structure parameters of an AMB, finally complete the parameterized modeling, as shown in Fig. 4.

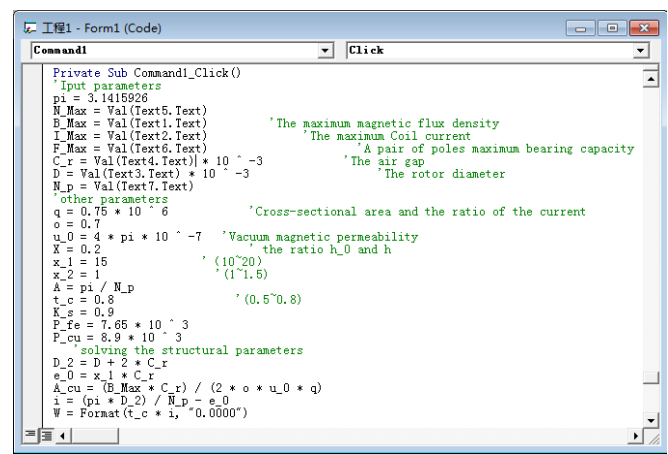

Figure 4. Code of the radial magnetic bearing on VB.

When users input or modify a set of design parameters, the parameterized model is directly generated, as shown in Fig. 5.

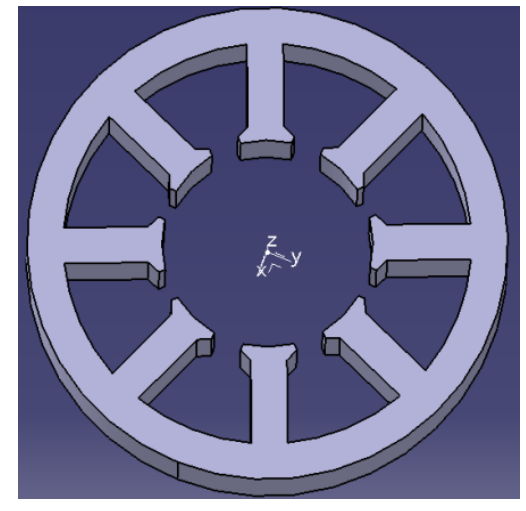

Figure 5. 3D model of the radial magnetic bearing.

\section{Conclusion}

The structure of active magnetic bearing is complex, its performance is influenced by structure parameters, electromagnetic parameters and control parameters. By adopting the method of parametric design, custom application driven magnetic bearing a parameterized model is built up, greatly improving the design efficiency, saving the cost. Assembly of magnetic bearing system integration is of great significance.

\section{References}

1. G. Schweitzer, H. Maslen. Magnetic bearing-Theory, design, and application to rational machinery, (2009).

2. A. Filatov, P. McMullen, L. Hawkins, E. Blumber. Magnetic Bearing Actuator Design for a Gas Expander Generator. Proceedings of the Ninth 
International Symposium on Magnetic Bearings, Lexington, KY, USA, (2004).

3. Yang jing, Gen Haipeng, Yu lie. Dynamic properties investigation about conical AMB. Proceeding of the eighth International Symposium on Magnetic Bearings, Mito, Japan, (2002).
4. C. Nataraj, E. Calver. Optional design of radial magnetic bearings. Proceeding of the sixth International Symposiuk on Magnetic Bearings, MIT, USA, (1998).

5. Xie Yuefeng, YU Xiongqing. Parametric design of aircraft configuration using API in CATIA. Computer Engineering and Design, 29,14 (2008). 